To appear in Connection Science

Vol. 00, No. 00, Month 20XX, 1-5

This is an Accepted Manuscript of an article published by Taylor \& Francis in Connection Science on 30 May 2017, available online: http://www.tandfonline.com/10.1080/09540091.2017.1323456.

\title{
Analysis of the EPSRC Principles of Robotics in Regard to Key Research Topics
}

\author{
A. Gning*, D. N. Davis, Y. Cheng and P. Robinson \\ University of Hull, Cottingham Road, Hull, HU6 7RX, UK \\ (v2.0 released January 2017)
}

\begin{abstract}
In this paper, we review the five rules published in EPSRC Principles of Robotics with a specific focus on future robotics research topics. It is demonstrated through a pictorial representation of the five rules that these rules are questionably not sufficient, overlapping and not explicitly reflecting the true challenges of robotics ethics in relation to the future of robotics research.
\end{abstract}

Keywords: The EPSRC Principles of Robotics, Robotics ethic, Behaviour traceability, Learning capabilities, ability limits, Humanoid Robots.

\section{Introduction}

In the modern world with the spectacular development of technological resources, robotics have resulted in numerous applications (Bekey et al., 2008) and often unpredicted deployment in real life (e.g. the increasing use of drones in civil applications). To go along with this age of robotics, the research community and the society in general need to define ethical principles that are general enough to be robust to evolutions in time and to be adapted to the range of possible applications and research. Ethical principles should be comprehensible and universalised enough for the designer and makers of robots to be selfaware of the regulations restrictions. In general, robotics applications can be classified into four groups: domestics or human assistive robots (Dautenhahn et al., 2005; Forlizzi \& DiSalvo, 2006; Sung, Grinter, Christensen, \& Guo, 2008), medical robots (Cleary \& Nguyen, 2001; Kwakkel, Kollen, \& Krebs, 2007; Moustris, Hiridis, Deliparaschos, \& Konstantinidis, 2011; Rassweiler, Binder, \& Frede, 2001), defence robots (Adams, 2001; Singer, 2008) and industrial robots (Bi, Lang, Shen, \& Wang, 2008; Leitão, 2009). In this paper, the discussion is focused on the first three groups of robots since the industrial robots are ethically less challenging (e.g. bounded to limited areas with pre-specified sets of limited tasks and are not in direct physical interaction with society). Industrial robots do raise a crucial debate in the risk of favouring unemployment but we choose to skip this point in this paper.

Five years ago in the UK, a panel of distinguished robotics and AI experts published the EPSRC Principles of Robotics in the form of five rules and seven high level messages. The contribution of this paper is to discuss these principles as research users. Our focus is on a transversal structure of robotics and ethics between: robotics subgroups, robotics

\footnotetext{
${ }^{*}$ Corresponding author. Email: e.gning@hull.ac.uk
} 
research topics and law frameworks. It is shown how the issue of ethics and robotics can be seen in a prism of this transversal structure. Additionally, a pictorial representation of the rules is presented and an analysis is given with respect to the three criteria of validity, sufficiency and utility.

The paper is organised as fellows. Section 2.1 shows the transversal structure when brainstorming ethics and the future of robotics. Then, Section 2.3 presents a pictorial description of the 5 rules and provides a discussion on the three criteria above.

\section{Discussion Over the Set of Five Rules}

\subsection{Transversal Structure of Robotics Ethics Through Law, Research Topics and Robotics Groups}

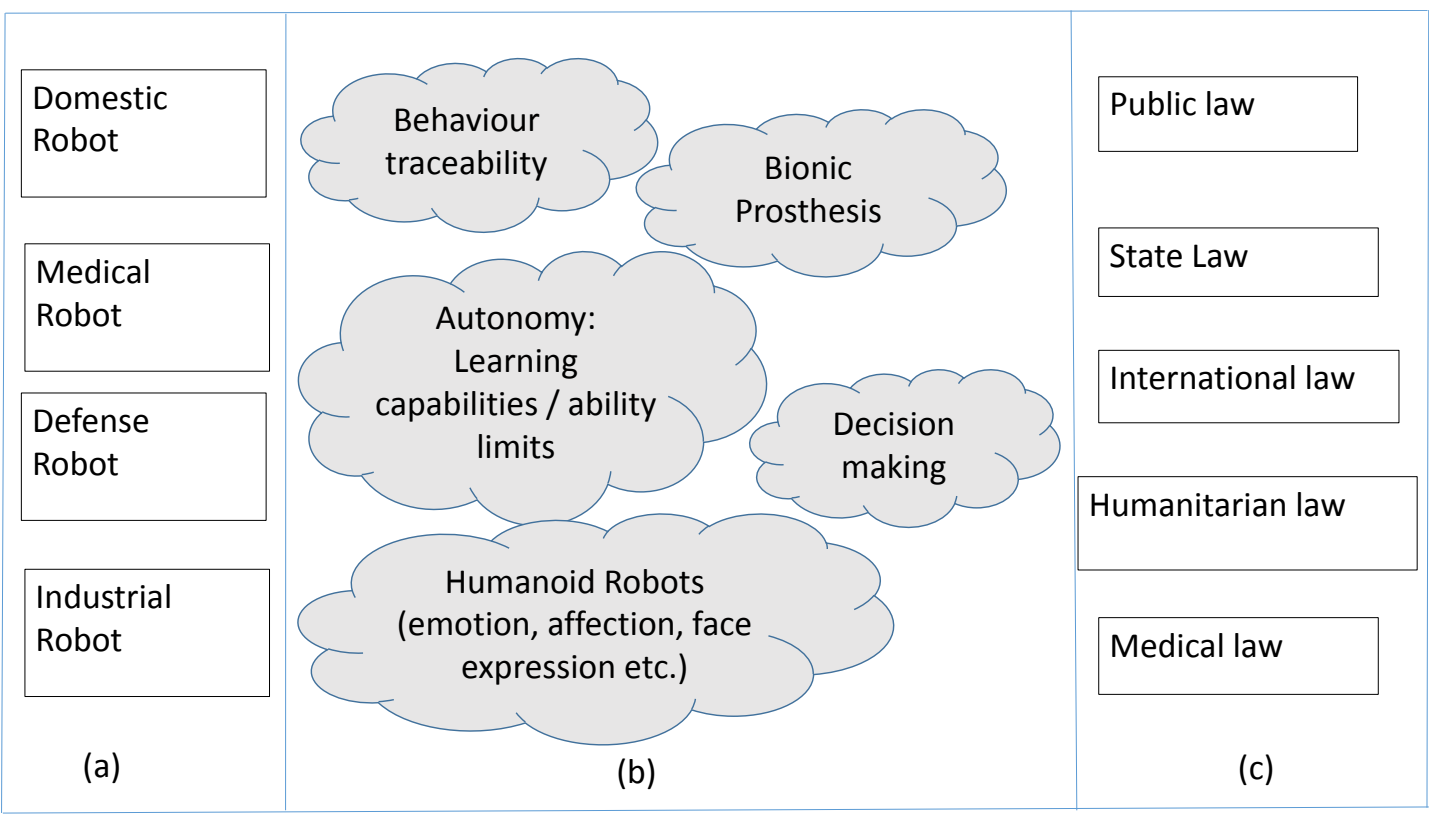

Figure 1. (a) robotics groups, (b) key area of robotics research topics raising ethics issues, (c) domain of law regulations to be considered.

Figure 1 lists the difficult task of regulating ethics for different group of robotics in regard to five popular research topics. In each group of robotics (figure 1-(a)), ethical questions can be raised and key ones are represented (figure 1-(b)).

- How can we design robots and be able to interpret their behaviour, to know about learning robots limits and boundaries.

- Humanoid robots with imitation of human gestures and face animation can push the interaction with human (especially children and disabled people) to new limits raising needs to regulate and anticipate on possible implications.

- These days, humans can greatly benefit from prosthesis thanks to progress on robotics research. However this can potentially result in non-disabled people seeking prostheses that can enhance their capabilities raising the need for new regulations.

- Finally, the most important questions relate to decision capabilities of robots. These decisions directly involve human life and hence raise questions of legal issues resulting from executed actions. 
On top of these research topics, robotics ethics have to be compatible with a set of law domains (figure 1(c)). There is a need to take into account that robots need to be compatible with different levels of laws that can change, for instance from region to region or state to state.

\section{2. $\quad$ Set of Five Rules}

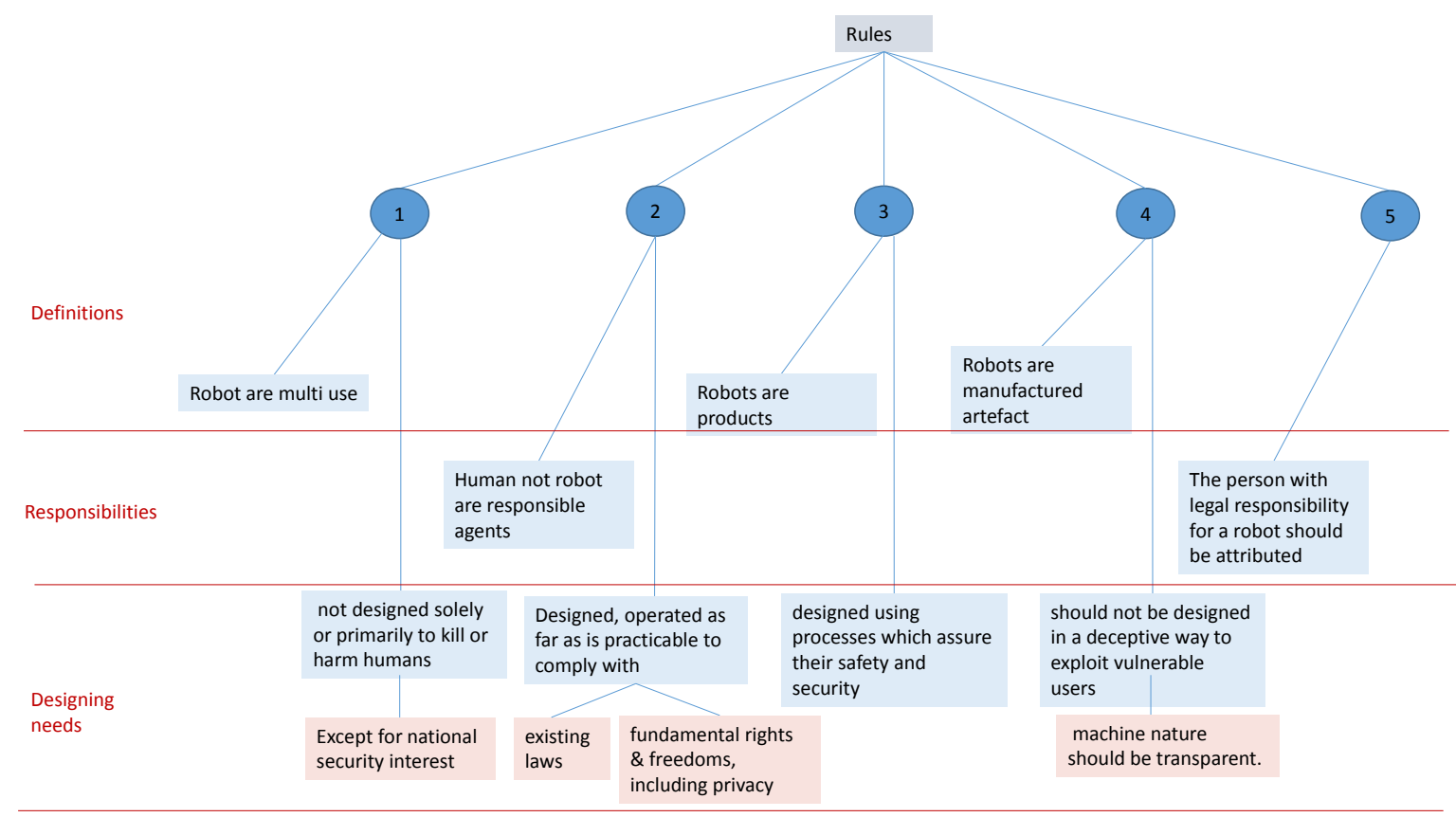

Figure 2. The set of five rules.

Figure 2 represents in a graphical way the set of five rules. It can be seen that there is a common pattern to the five rules: the first sentences are often generalities and definitions about robots such as "robots are products" or state that the responsibilities are not liable to robot but human instead. The last sentences state the designing needs and bounce between safety issues, legal issues and transparency issues.

\subsection{Discussion}

In regard to section 2.1, a first comment that can be made about the set of five rules is that it is ambitious to think that it is possible to give common/uniform principles to all types of robots, across all possible evolutions of research and all frameworks of law. It would, perhaps, be more natural to seek guidance rules reflecting the transversal nature of robotics shown in figure 1. Principles, and guidances for each research group may be a more realistic approach.

Consequently, we believe that the five rules in the principles are not sufficient and that the principle should be specified and particularised for each group of robot in figure 1-(a) taking into account the specific aspect of laws and research avenues involved in figure 1(c) and (b). Discussion, criticisms and questions in relation to the graphical structure 
shown in Figure 2 can be listed in the following subsections

\subsubsection{Overlapping rules?}

The five rules are sometime overlapping. For instance, looking at the graph in figure 2, "complying with existing law" in rule\#2 encapsulates "not designed solely or primarily to kill or harm human" in rule\#1. In other words, if robots are operated to comply with existing law, logically, they should not also be designed solely or primarily to kill or harm human. The novelty in rule\#1 is rather an exception occurring when national security are of interest. Consequently, would it not be more relevant to have, for example in rule\#1 that "robots should comply with existing law with exception to military/defense applications where robot can be designed solely or primarily to kill or harm human"?

Another example is "the person with legal responsibility for a robot should be attributed" in rule\#5 can encapsulate implicitly "human not robot are responsible agents" in rule\#2. In other words, it can be argued that in rule\#5 "responsibility for a robot should be" imply that robot are not responsible.

Fundamentally, these overlapping and repetitive assertions in the rules are not wrong. However, as a good practice and ideally, the five rules should cover different principles. In that sense this analysis using the graphical representation is a useful exercise.

\subsubsection{Sufficiency?}

The five rules are not sufficient in the sense that there are not inclusive of all ethical challenges that will potentially be raised in the future. For example, we can barely see how Bionic Prosthesis can be fitted in the rules at the present form (e.g., robotics research can allow in the future modification of human body in order to gain more power, speed etc.). Artificial Sexuality is another example of controversial research that can result into ethical questions. Although deception is covered in the principles, the reaction of the society toward artificial sexuality is another challenging issue. What are the limits? who can use it? what are the consequences? if we draw parallels to the debate on pornography, ethics become difficult to define for artificial sexuality.

Additionally, the five rules are not explicitly considering that law can be contradictory depending on the domain considered from councils, regions, countries and continent. A similar example often encountered in research is patent applications for which several specific studies are needed to apply in given regions of the world. Laws can be even more complicated since religious belief and people habits and customs will dictate the notion of ethics.

\section{Conclusion}

In this paper, we provided a short discussion on the EPSRC Principles of Robotics in relation to research topics. Through a pictorial representation of the five rules, it is questioned that these rules are not in fact sufficient, overlap and do not explicitly reflect the true challenges of robotics ethics. We based part of our reasoning on the transversal nature of robotic ethics along three lines: groups that constitutes robotics, future avenues of robotics that are essential to be captured when defining ethics, and the structured nature of law.

As a conclusion we recommend a formulation that will differentiate ethics for each group of robotics while being more aware of the contradictions and strong constraints 
that can exist due to the structural nature of law.

\section{References}

\section{References}

Adams, T. K. (2001). Future warfare and the decline of human decisionmaking. Parameters, $31(4), 57$.

Bekey, G., Ambrose, R., Kumar, V., Lavery, D., Sanderson, A., Wilcox, B., ... Zheng, Y. (2008). Robotics: State of the art and future challenges. World Scientific.

Bi, Z. M., Lang, S. Y., Shen, W., \& Wang, L. (2008). Reconfigurable manufacturing systems: the state of the art. International Journal of Production Research, 46(4), 967-992.

Cleary, K., \& Nguyen, C. (2001). State of the art in surgical robotics: clinical applications and technology challenges. Computer Aided Surgery, 6(6), 312-328.

Dautenhahn, K., Woods, S., Kaouri, C., Walters, M. L., Koay, K. L., \& Werry, I. (2005). What is a robot companion - friend, assistant or butler. In In proc. ieee iros (pp. 1488-1493).

Forlizzi, J., \& DiSalvo, C. (2006). Service robots in the domestic environment: A study of the roomba vacuum in the home. In Proceedings of the 1st acm sigchi/sigart conference on human-robot interaction (p. 258-265). New York, NY, USA: ACM. Retrieved from http://doi.acm.org/10.1145/1121241.1121286 doi:

Kwakkel, G., Kollen, B. J., \& Krebs, H. I. (2007). Effects of robot-assisted therapy on upper limb recovery after stroke: a systematic review. Neurorehabilitation and neural repair.

Leitão, P. (2009). Agent-based distributed manufacturing control: A state-of-the-art survey. Engineering Applications of Artificial Intelligence, 22(7), 979-991.

Moustris, G. P., Hiridis, S. C., Deliparaschos, K. M., \& Konstantinidis, K. M. (2011). Evolution of autonomous and semi-autonomous robotic surgical systems: a review of the literature. The International Journal of Medical Robotics and Computer Assisted Surgery, 7(4), 375-392. Retrieved 2012-09-01, from http://onlinelibrary.wiley.com/doi/10.1002/rcs.408/abstract doi:

Rassweiler, J., Binder, J., \& Frede, T. (2001). Robotic and telesurgery: will they change our future? Current opinion in Urology, 11(3), 309-320.

Singer, P. (2008). Robots at war. Wilson Quarterly.

Sung, J.-Y., Grinter, R. E., Christensen, H. I., \& Guo, L. (2008, March). Housewives or technophiles?: understanding domestic robot owners. In Hri-08: Proc of $3 \mathrm{rd} \mathrm{acm/ieee}$ intl conf on human robot interaction. Amsterdam. 